\title{
LEADING EDGE CAVITATION IN A CENTRIFUGAL PUMP: NUMERICAL PREDICTIONS COMPARED WITH MODEL TESTS
}

\author{
R. HIRSCHI, PH. DUPONT AND F. AVELLAN \\ IMHEF-EPFL \\ 33, av. de Cour, CH-1007 Lausanne, Switzerland \\ E-mail: roland.hirschi@imhef.dgm.epfl.ch \\ AND \\ J.-N. FAVRE, J.-F. GUELICH AND W. HANDLOSER \\ Sulzer Pumps P.O. Box 65 \\ 8404 Winterthur, Switzerland
}

\begin{abstract}
The aim of this paper is to present the results obtained with a 3-D numerical method allowing the prediction of the cavitation behaviour of a centrifugal pump and to compare this prediction to model tests.

The proposed method consists in assuming the cavity interface as a free surface boundary of the computation domain and in computing the single phase flow. The unknown shape of the interface is predicted by an iterative procedure matching the cavity surface to a constant pressure boundary $\left(p_{v}\right)$. The originality of the presented method is that the adaptation process is done apart from the flow calculation, allowing to use any available code.
\end{abstract}

\section{Introduction}

Cavitation behaviour prediction of hydraulic machines, such as inception $\psi_{c_{i}}$ and standard $\psi_{c_{s}}$ cavitation coefficients, partial cavity length and its associated performance drop is of high interest for the manufacturers.

When upgrading existing hydraulic installations or designing new geometries, the cavitation guarantees are often the main limiting features. A precise prediction of this phenomenon by numerical simulation is then essential. In recent years, models to predict cavitation development have been refined and applied with success on isolated profiles, for steady [3] and unsteady flows[2][7]. Some 3-D models, based on S1/S2 and Euler flow computation were also developed, but without taking into account the 3-D turbulent and viscous effects of the cavity on the flow [8].

To present the accuracy of the new proposed method, numerical calculations are performed on a centrifugal pump with a commercial Navier-Stokes code (TASCflow). A first analysis enables an intrinsic verification of the numerical results given by the solver and make sure they are physically meaningful. Once this analysis is done, particular operating points are chosen to predict the impeller cavitation behaviour. 


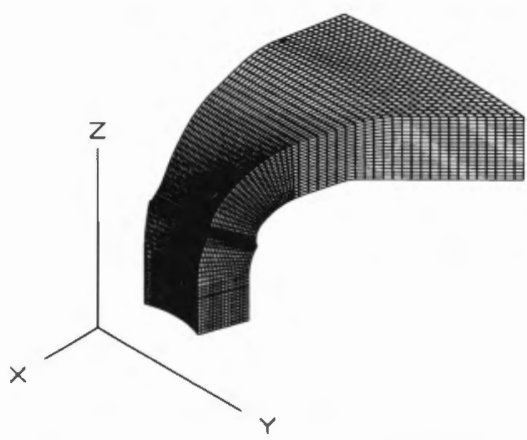

Figure 1. 3D view of the inlet pipe mesh

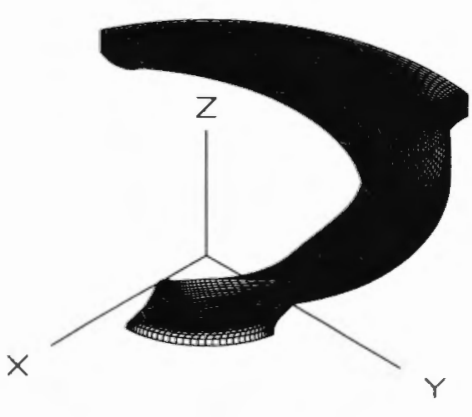

Figure 2. 3D view of the impeller mesh

Cavitation free flow computation is performed, in order to define the hydraulic performances and the cavitation inception coefficient $\psi_{c_{i}}$, which is predicted using the minimum pressure coefficient calculated over the blade. Then, the cavity lengths as well as the initial cavity shapes are established as a function of the cavitation coefficient using the Rayleigh-Plesset equation. Finally, the cavitation coefficient $\psi_{c_{s}}$, corresponding to the beginning of the head drop, is determined by a direct computation of the pump flow, taking into account the flow modification due to the cavitation sheet. The comparison with the measurements is done defining a local cavitation coefficient, based on the $\psi_{c}$ cavitation coefficient measured during the model tests [1] [4].

The cavitation behaviour prediction of the impeller is only possible if the flow field at its inlet is well known. For that reason, the inlet pipe flow computation is performed in order to define the velocity field and the evolution of the absolute flow angle at its outlet. In Figure 1 is represented the inlet pipe mesh and in Figure 3 the flow field characteristics at its outlet. The Figure 3 brings to the fore the quality of the resulting absolute flow angle repartition. The angular variations in comparison with the ideal angle of 90 degrees are lower than 0.1 degree. Considering these results, one can assume the velocity field to be axial at the outlet of the inlet pipe. This condition will be used as an inlet condition of the impeller flow computation.

\section{Single phase flow computation}

Eight operating points are calculated using a Reynolds averaged Navier \& Stokes finite volume code. The turbulence is taking into account using the well known $k-\varepsilon$ model. As the resolution of the near wall region would require a large number of nodes, a common approach is to model this region using "wall-function". This way allows to relate the near wall tangential velocity to the wall shear stress by means of a logarithmic relation.

The mesh of Figure 2 is a single structured bloc with 95 nodes in the flow direction, 41 from suction to pressure side and 17 from hub to shroud. 


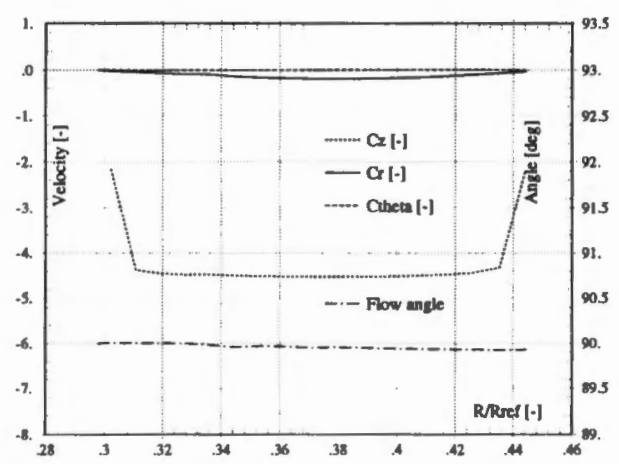

Figure 3. Velocity and absolute flow angle survey at the inlet pipe outlet

\section{Cavity length}

If one assumed that the dynamic behaviour of the cavity is similar to the bubble one, then the use of the Rayleigh-Plesset equation [6], which gives the evolution of a bubble radius depending of the pressure field acting around, allows to take into account the cavity dynamic behaviour. Especially, cavitation sheet beginning as well as its closure is relatively well predicted using the bubble explosion and collapse location. Previous experiments in a test section with a 2-D profile have shown this approach to be in most of the cases surprisingly accurate to predict these two points, even if the cavity thickness needs to be corrected.

\section{Initial cavity shape}

The proposed method used for the computation with the cavitation sheet has the great advantage to be independent of the flow calculation code. However, the iterative deformation process could be quite time consuming if beginning with the solid surface of the blade. In order to estimate an initial cavity shape, the Rayleigh-Plesset equation is used. As illustrated in Figure 4, the initial cavity shape is supposed to be the envelope of the bubble evolution.

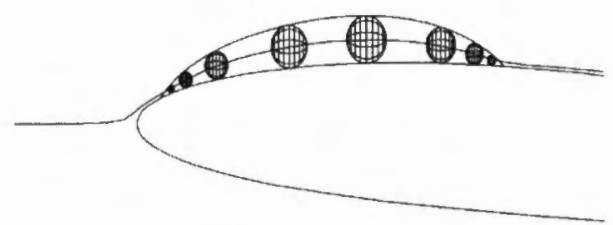

Figure 4. Initial cavity shape $=$ bubble evolution envelope 


\section{Deformation method}

Generally, for 2-D flow computations, one can observe small differences between the initial cavity shape obtained with the method described in the previous section and the deformed one (see Figure 5). But for 3-D problems, the deformation method is necessary in order to take into account the 3-D effects of the cavity on the flow.

So, the cavity shape adaptation is determined according to the pressure distribution obtained with the flow computation at the previous iteration. If one considers $t$ the subscript corresponding to the calculated values at the previous iteration, the expression of the modified cavity thickness $e$ at the abscissa $i$ is given by:

$$
e_{i}^{t+1}=e_{i}^{t}+C_{2}\left(c_{p_{i}}^{t}+\chi_{c}\right)
$$

where $C_{2}$ is a coefficient depending on the Reynolds number and a relaxation coefficient $C_{1}$.

$$
C_{2}=f\left(R_{e}\right) \frac{\left[2-2^{\left(1-C_{1}\right)}\right]}{1+\left|\frac{\partial^{2} e}{\partial s^{2}}\right|_{i}^{t}} \quad \text { and } \quad C_{1}= \begin{cases}1 & \text { if }\left|c_{p_{i-1}}+\chi_{c}\right|>S_{c p} \\ \frac{\left|c_{p_{i-1}}+\chi_{c}\right|}{S_{c p}} & \text { otherwise }\end{cases}
$$

The coefficient $\left|\frac{\partial^{2} e}{\partial s^{2}}\right|_{i}^{t}$ allows us to avoid oscillations in regions where high thickness gradients occurs, such as the closure of the cavity. $S_{c p}$ corresponds to the root mean square of the difference $\delta_{i}^{t}$ between the pressure coefficient at the abscissa $i$ and the local cavitation coefficient value $\chi_{c}$, on all cavity nodes, and is given by:

$$
S_{c p}=\sqrt{\frac{1}{f p-d p} \sum_{i=d p}^{i=f p}\left(\delta_{i}^{t}-\bar{\delta}^{t}\right)^{2}} \quad \text { and } \quad \delta_{i}^{t}=c_{p_{i}}^{t}+\chi_{c}
$$

with $\bar{\delta}^{t}$ the average value of $\delta_{i}^{t}$ on all cavity points. The cavity beginning does not always correspond to the leading edge, so the deformation starts at the abscissa $i$ where the following condition is respected:

$$
\left|\frac{\partial^{2} e}{\partial s^{2}}\right|_{i}^{t}>1
$$

The Figure 5 shows an application of this method on a 2D profile.

\section{Results}

\subsection{CAVITATION INCEPTION}

The cavitation inception condition, in terms of local cavitation coefficient and static pressure, is given by the condition $\chi_{c} \leq-C p_{\min }$ where $C p_{\min }$ represents the minimum value of the calculated static pressure coefficient in the impeller [5]. Non dimensionalized, this condition is given by: 


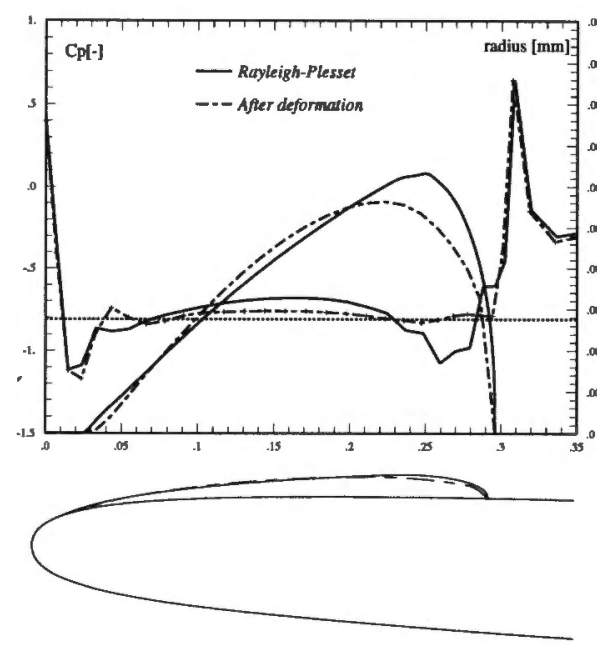

Figure 5. Cavity deformation on a 2D profile

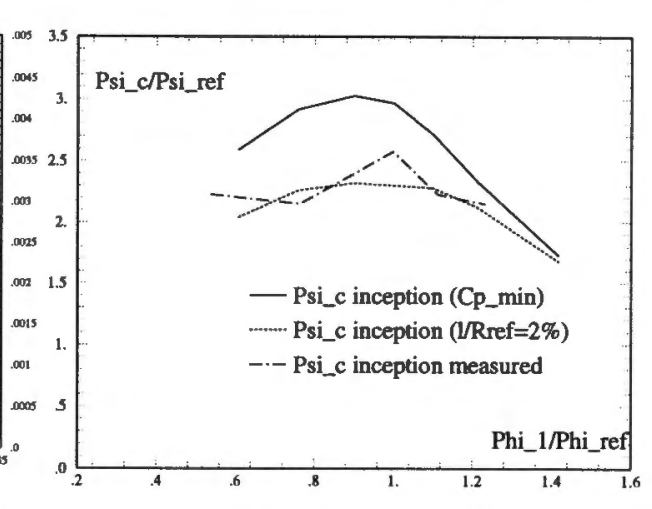

Figure 6. Cavitation inception prediction compared with measurements

$$
\psi_{c} \leq-C p_{\min }+\left[1+k_{r d}\right] \frac{R_{1}^{4} A_{1}^{2}}{R_{1}^{4} A_{\overline{1}}^{2}} \phi_{1}^{2}
$$

On the Figure 6 are compared the cavitation inception curve obtained with the minimum pressure coefficient calculated, the same curve obtained with the cavitation inception criterion defined as a cavity length equal to $2 \%$ of the outlet radius of the impeller and the measurements curve obtained with this same criterion. These results show that the measurements results are very well predicted by this method. A other point to notice, especially in such a pump of a small specific speed, is the leakage flow between the impeller and the casing on shroud side: an accurate estimation of this leakage flow, in order to calibrate the real flow inside the impeller, is essential for an useful comparison.

\subsection{CAVITY LENGTH}

Out of the shock less entry, which corresponds to zero incidence, the $\left|C p_{\min }\right|$ increases on the suction side for low flow rate or on the pressure side for high flow rate. The direct consequence is a cavity inception and development. In Figure 7 are reported the calculated cavity lengths on five streamlines, from hub to shroud, for four operating points. Remember that the cavity length corresponds to the bubble collapse calculated by the Rayleigh-Plesset equation. The comparison with the measurements, corresponding to the square points, shows the assumption made in paragraph 4 (cavitation sheet dynamic behaviour = bubble dynamic behaviour) to be valid. 

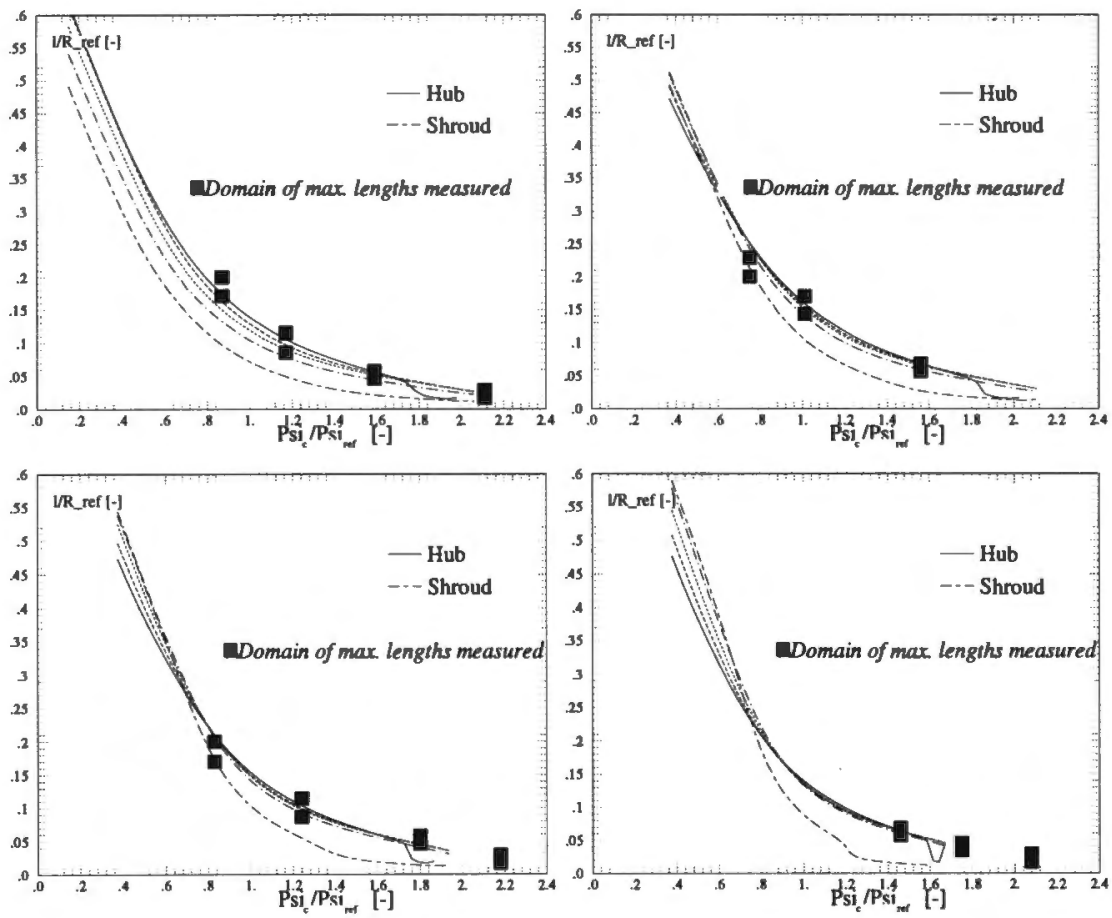

Figure 7. Predicted cavity lengths compared with measurements

\subsection{CAVITY DEFORMATION}

Once the initial cavity determined, its shape is iteratively modified until the calculated pressure at its interface is constant and equal to the vapor pressure. The pressure coefficient distributions (grid lines $2=$ hub, 9, $13 \&$ $16=$ shroud) obtained after the flow computation without cavitation and for a $\psi_{c} / \psi_{\text {ref }}$ value of 0.265 are compared in Figure 8 . The cavity effect on the pressure distribution corresponds to the constant pressure region on the suction side. In this example, the pressure loss at the leading edge is partially compensated by the pressure increase near the cavity closure region.

\subsection{HEAD DROP COMPUTATION}

The comparison of the torque, obtained by the pressure and the viscous forces integration on the blades and impeller side walls, to the moment of momentum in the relative frame of reference allows to quantify the energy loss due to the cavity. In term of torque this is expressed by:

$$
\vec{T}_{t}=\vec{T}_{f}+\vec{T}_{r}
$$

Where $\vec{T}_{t}$ represents the transferred torque, $\vec{T}_{f}$ the provided torque and $\vec{T}_{r}$ the loosed torque. 

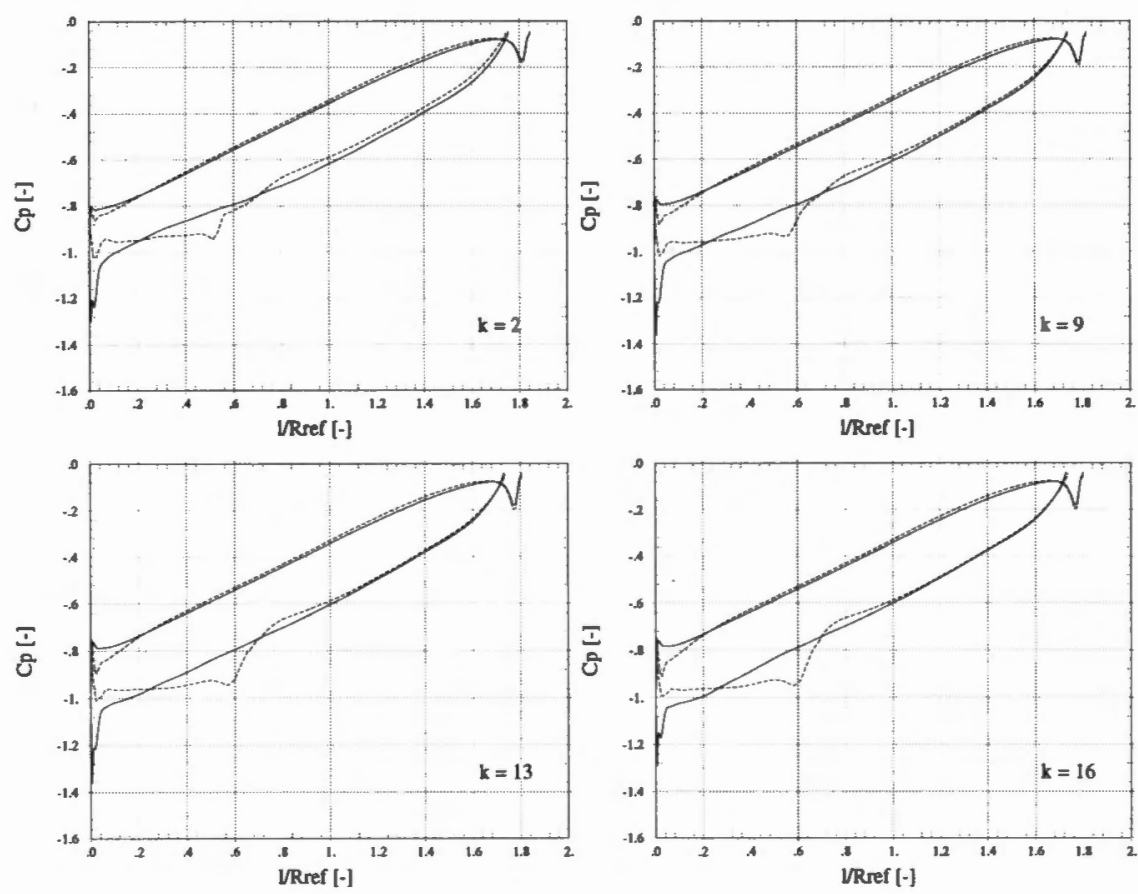

Figure 8. Pressure coefficient distribution without cavitation and for $\psi_{c} / \psi_{\text {ref }}=0.265: \phi_{1} / \phi_{\text {ref }}=1.21$

Transferred power The transferred torque given by the impeller is:

$$
\vec{T}_{t}=\int_{S_{i m p}} \vec{r} \wedge(p \vec{n}) d s+\int_{S_{i m p}} \vec{r} \wedge(\overline{\bar{\tau}} \vec{n}) d s
$$

where $S_{i m p}$ represents the blades and impeller side walls, $p$ the calculated pressure, $\vec{r}$ the position vector and $\overline{\bar{\tau}}$ the constraints tensor, which correspond to the molecular and turbulent viscosity. The transferred energy can be expressed under a non dimensional number $\psi_{t}$ through the transferred power:

$$
P_{t}=\vec{T}_{t} \cdot \vec{\Omega}=\rho Q E_{t} \quad \rightarrow \quad \psi_{t}=\frac{2 E_{t}}{\omega^{2} R_{1}^{2}}
$$

Provided hydraulic power The expression of the provided power is given by the moment of momentum equation in the relative frame of reference. Let $\vec{T}_{f}$ to be the torque representative of the provided moment of momentum:

$$
\vec{T}_{f}=-\int_{S_{t o t}} \vec{r} \wedge(\rho \vec{w}) \vec{w} \vec{n} d s+\int_{V} \vec{r} \wedge(\rho \vec{S}) d v+\int_{S_{t o t}} \vec{r} \wedge(\overline{\bar{\tau}} \vec{n}) d s
$$

where $\vec{S}$ corresponds to the centrifugal and Coriolis effects, and where $S_{t o t}$ represents all the domain surfaces, delimited by the inlet and outlet of the 
impeller. It is then possible to express the provided energy in the form:

$$
P_{f}=\vec{T}_{f} \cdot \vec{\Omega}=\rho Q E_{f} \quad \rightarrow \quad \psi_{f}=\frac{2 E_{f}}{\omega^{2} R_{1}^{2}}
$$

In the Table 1 are reported, for three relative $\psi_{c}$ values, the energy coeffcient representative of the provided energy $\left(\psi_{f}\right)$ and the transferred energy $\left(\psi_{t}\right)$. For a quite constant transferred energy value, one remarks that the provided energy is decreasing with the pressure level $\psi_{c}$ at the impeller inlet section. If one plots the calculated relative head evolution, which corresponds to the provided energy, versus the $\psi_{c}$ value (Figure 9), it can be noticed that the $\psi_{c_{s}}$, which corresponds to the head drop beginning, coincides very well with the measured one. The results exposed in Figure 9 show that the calculated impeller head is over-estimated in comparison with the measurements. However, it has to be taken into account the fact that the presented measurements are representative of the entire pump, with the fixed parts (diffuser, inlet pipe,..). It is then reasonable to think that this over-estimation is mainly due to the losses in the fixed parts of the machine, which are not taking into account by the computation.

\begin{tabular}{lll}
\hline$\psi_{c} / \psi_{\text {ref }}$ & $\psi_{f}[-]$ & $\psi_{t}[-]$ \\
\hline cav. free. & 1.129 & 1.28 \\
0.383 & 1.12 & 1.285 \\
0.265 & 1.1 & 1.28 \\
\hline
\end{tabular}

TABLE 1. Impeller provided and transferred energy as a function of $\psi_{c}\left(\phi_{1} / \phi_{\text {ref }}=1.21\right)$

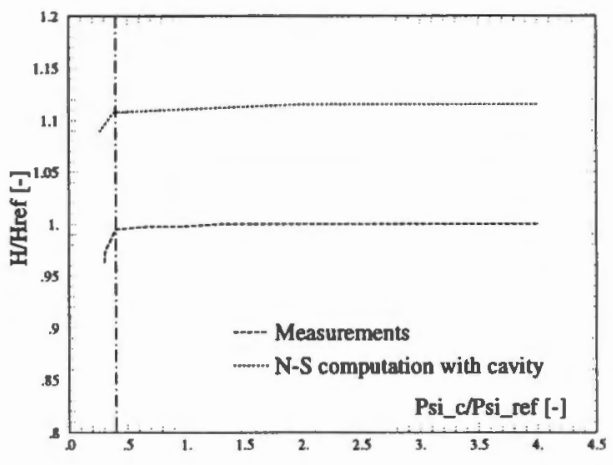

Figure 9. Predicted and measured head drop

\section{Conclusion}

Considering an impeller geometry, its behaviour and its main cavitation characteristics can be described with the results of a Navier-Stokes 3-D computation, using models of cavitation developments. The predicted behaviour of the cavitation flow in a centrifugal pump, using the proposed method, compares very well with the measurements.

It was brought to the for that the knowledge of the impeller inlet velocity field is essential for the cavitation behaviour prediction. Therefore, a flow computation of the inlet pipe was first performed.

The pressure level corresponding to cavitation inception is determined for all calculated operating points and compared with measurements. The mean standard deviation between the measured and calculated points is 
lower than $4 \%$.

The cavity lengths computation using the Rayleigh-Plesset equation provides very good results. Indeed, the measured cavity lengths behaviour is faithfully reproduced by the calculation, and this for all measured operating points.

The prediction of the head drop beginning is done by a direct flow computation taking into account the cavitation sheet. The very good results obtained with this method proves its efficiency to predict head drop due to cavitation. As a conclusion, the results analysis of this study demonstrates that the presented method provides an interesting tool for the 3-D cavitation development prediction in hydraulic machines.

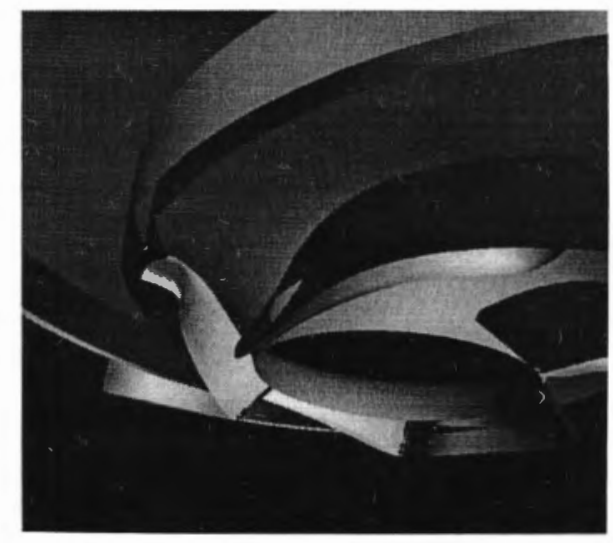

Figure 10. 3D view of the cavitation sheet

\section{Acknowledgments}

The authors are particularly grateful to the members of the IMHEF Cavitation research group. We wish to thank SULZER Pumps which made possible the publication of this paper and the staff of the test-rig who performed the measurements. This work is financially supported by the Swiss Federal "Commission d'Encouragement à la Recherche Scientifique", SULZER Pumps, Hydro-Vevey S.A. and Rolla S.P. Propeller.

\section{Nomenclature}

$\begin{array}{lll}e & : & \text { thickness } \\ k_{r d} & : \text { coefficient of viscous losses } \\ p & : \text { static pressure } \\ p_{v} & : \text { vapor pressure } \\ C p & : \text { pressure coefficient } \\ E & : \text { energy } \\ H & : \text { head } \\ P & : \text { power }\end{array}$




$\begin{array}{lll}Q & : \text { flow rate } \\ R & : \text { impeller radius } \\ S, A & : \text { area } \\ T & : \text { torque } \\ V & : \text { volume of the delimited domaine } \\ \vec{w} & : \text { relative velocity } \\ \vec{r} & : \text { position vector } \\ \vec{n} & : \text { normalized vector } \\ \vec{\Omega} & : \text { vector of the angular rotation speed } \\ \alpha & : \text { absolute flow angle } \\ \omega & : \text { angular rotation speed } \\ \rho & : \text { density } \\ \chi_{c} & : \text { local cavitation coefficient at impeller inlet } \\ \phi_{1} & : \text { flow rate coefficient } \\ \phi_{\text {ref }} & : \text { esign flow rate coefficient } \\ \psi_{1} & : \text { design energy coefficient } \\ \psi_{\text {ref }} & : \text { net positive suction energy coefficient } \\ \psi_{c} & \end{array}$

$$
\begin{aligned}
& \left(m^{3} / s\right) \\
& (m) \\
& \left(m^{2}\right) \\
& (N m) \\
& \left(m^{3}\right) \\
& (m / s) \\
& (m) \\
& (m / s) \\
& \left(s^{-1}\right) \\
& \left({ }^{\circ}\right) \\
& \left(s^{-1}\right) \\
& \left(k g / m^{3}\right) \\
& \chi_{c}=\frac{p_{1}-p_{v}}{\frac{1}{2} \rho U_{1}^{2}} \\
& \phi_{1}=\frac{Q}{\pi R_{1}^{3}} \\
& \psi_{1}=\frac{2 E}{\omega^{2} R_{1}^{2}} \\
& \psi_{c}=\frac{2 N P S E}{\omega^{2} R_{\frac{1}{1}}^{2}}
\end{aligned}
$$

\section{Subscripts}

$I$
$\overline{1}$
$\bar{I}$
$\bar{I}$
$i$
$t$

$$
\begin{aligned}
& \text { high pressure section of the machine (pump : volute outlet) } \\
& \text { high pressure section of the impeller (pump : impeller outlet) } \\
& \text { low pressure section of the impeller (pump : impeller inlet) } \\
& \text { low.pressure section of the machine (pump :inlet pipe inlet) } \\
& \text { reference to a particular streamline } \\
& \text { reference to a domain node in the flow direction } \\
& \text { reference to the previous time step }
\end{aligned}
$$

\section{References}

1. F. Avellan. Cavitation tests of hydraulic machines: Procedures and instrumentation. ASME Winter Ann. meeting, 1993.

2. Y. Delannoy and J.L. Kueny. Two phase flow approach in unsteady cavitation modeling. ASME, Toronto, 1990.

3. Ph. Dupont and F. Avellan. Numerical computation of leading egde cavity. ASME Summer Ann. meeting, 1991.

4. Ph. Dupont, E. Parkinson, and W. Walther. Cavitation development in a centrifugal pump: numerical and model tests predictions. ASME Winter Ann. meeting, 1993.

5. J.P. Franc, F. Avellan, B. Belahadji, L. Brianon-Marjollet J.Y. Billard, D. Frchou, A. Akarimi D.H. Fruman, J.L. Kueny, and J.M. Michel. La cavitation. Presses universitaires de Grenoble, 1995.

6. R.T. Knapp, J.T. Daily, and F.G. Hammit. Cavitation. Mac Graw Hill, New-York, 1970.

7. A. Kubota, H. Kato, and H. Yamaguchi. A new modelling of cavitating flows: a numerical study of unsteady cavitation on a hydrofoil section. J.F.E, 1992.

8. T. Maitre, J.L. Kueny, P. Geai, and A. Von Kaenel. Numerical prediction of threedimensionnal partial cavitation in a racket turbopump inducer. ASME Summer Ann. meeting, Washington, 1993. 